\title{
GLOBAL EXISTENCE AND QUENCHING FOR A DAMPED HYPERBOLIC MEMS EQUATION WITH THE FRINGING FIELD
}

\author{
TOSIYA MIYASITA
}

\begin{abstract}
We study a damped hyperbolic MEMS equation with the fringing field. It arises in the Micro-Electro Mechanical System(MEMS) devices. We give some criteria for global existence and quenching of the solution. First we establish a time-local solution by a contraction mapping theorem. This procedure is standard. Next we show that there exists a global solution for the small parameter and initial value. Finally, we deal with the quenching result for the large parameter.
\end{abstract}

\section{Introduction}

We consider the following damped hyperbolic MEMS equation with the fringing field:

$$
\begin{cases}u_{t t}+u_{t}=u_{x x}+\lambda \frac{1+\delta\left|u_{x}\right|^{2}}{(1-u)^{2}} & x \in I, t \in(0, T), \\ u(0, t)=u(1, t)=0 & t \in(0, T), \\ u(x, 0)=u_{0}(x) & x \in I, \\ u_{t}(x, 0)=u_{1}(x) & x \in I,\end{cases}
$$

where $\lambda>0, \delta>0, I=(0,1)$ and $0 \leq u_{0}<1$. (1) arises in the study of the Micro-Electro Mechanical System(MEMS) devices. Here $\lambda$ denotes the voltage and $\delta\left|u_{x}\right|^{2}$ is called the fringing field $[29,35]$. The MEMS devices are often utilized to combine electronics with micro-size mechanical devices. The MEMS devices can be modelled as the dynamic deflection of an elastic membrane inside this system and arise in the accelerometers for airbag deployment in automobiles, in the ink jet printer heads, in the optical switches, in the chemical sensors and so on. For more details, see $[8,9,34]$ and references therein. If the solution $u(x, t)$ of (1) reaches 1 at some point in $\bar{I}$ in finite time $t=T_{q}$, the right-hand side becomes infinite, which leads to the singularity. In this case, the solution $u(x, t)$ is said to quench in finite time $t=T_{q}$ and $T_{q}$ is called the quenching time of the solution. Let $A u$ be an elliptic $-\Delta u$, parabolic $u_{t}-\Delta u$, hyperbolic $u_{t t}-\Delta u$ or damped hyperbolic operator $u_{t t}+u_{t}-\Delta u$ with Dirichlet boundary

Received February 14, 2016, accepted June 4, 2016.

2010 Mathematics Subject Classification. 35L70, 74H35, 74H40, 74K15.

Key words and phrases. MEMS, damped hyperbolic, fringing field, local solution, global solution, quenching. 
condition on the general higher dimensional domain $\Omega$. If $\delta=0$, we have many results of stationary problem, time-global existence and quenching in finite time. On the contrary, the problem with the fringing field

$$
A u=\lambda \frac{1+\delta|\nabla u|^{2}}{(1-u)^{2}},
$$

is not studied enough. For an elliptic operator $A$, there are results of minimal solution, its stability and the bifurcation diagram in $[4,10,40]$. They obtain the upper bound $\lambda^{*}$ of the existence of the stationary solution for fixed $\delta>0 . \lambda^{*}$ is called the pull-in voltage. For a parabolic operator $A$, in [30,39], they prove that the solution exists globally in time for $\lambda \leq$ $\lambda^{*}$ and that quenches in finite time for $\lambda>\lambda^{*}$. Moreover they consider the location of the quenching point in [30] and the estimate of the quenching time in [39]. However, it seems that there are no studies for a hyperbolic and damped hyperbolic operator $A$. The aim of this paper is to consider the global existence and quenching of the solution for the damped hyperbolic equation (1) with the fringing field. We introduce the related results and consider the effects of the fringing field. First the problem

$$
A u=\lambda \frac{f(x)}{(1-u)^{p}},
$$

is considered, where $p>1$ and $f(x)=1$ or $f(x)=|x|^{\beta}$ for $\beta \geq 0$. For an elliptic and parabolic operator $A$, thanks to the maximum principle, we have the results of the time-global existence $[12,19,23,25]$ for sufficiently small $\lambda>0$, the quenching [12, 18, 23, 25] for sufficiently large $\lambda>0$, the connecting orbit [23], the Morse-Smale property [23], the location of the quenching point [17] and its stationary solution [5, 6, 7, 11, 13, 23]. Also in the hyperbolic problem, we have similar results to those in the parabolic case, i.e., the global existence [3, 26, 38], the quenching [3, 26, 32, 38], the estimate of the quenching time [32] and the singularity of the derivative [2]. In the damped hyperbolic case, we have the global existence [27] and quenching $[15,27]$. Next recently the nonlocal problem

$$
A u=\lambda \frac{1}{(1-u)^{2}\left(1+\int_{\Omega} \frac{d x}{1-u}\right)^{2}},
$$

is studied intensively. Although we can not apply the comparison principle owing to the nonlocal term, in the elliptic and parabolic problem, there are similar results to those without nonlocal term, i.e., the global existence [14, 16, 20], its asymptotic behaviour [20], the quenching $[14,16,20]$ and its stationary solution $[14,16,20,22,33,36]$. For a hyperbolic and damped hyperbolic operator $A$, we have the results of the global existence [15, 22] and the quenching [22] for $\Omega=(0,1)$. If we assume that the domain and the solution are radially symmetric, we have the global solution [31]. Lately, in [28], the authors derive the global solution for the higher dimensional domain $\Omega$ for a damped hyperbolic operator $A$.

The first theorem is concerned with the local existence of the solution. 
Theorem 1. Let $X \equiv H^{2}(I) \cap H_{0}^{1}(I), D \equiv X \times H_{0}^{1}(I)$ and $H \equiv H_{0}^{1}(I) \times L^{2}(I)$. For any $\lambda>0, \delta>0$ and $\left(u_{0}, u_{1}\right) \in D$ with

$$
\left\|u_{0}\right\|_{X} \leq \frac{1-\theta}{C_{S}},
$$

for $0<\theta<1$, there exists a unique solution of (1) with

$$
\phi=\left(\begin{array}{c}
u \\
u_{t}
\end{array}\right) \in C([0, T) ; D) \cap C^{1}([0, T) ; H),
$$

for sufficiently small $T>0$. The solution $u$ can be continued as long as $\|u(\cdot, t)\|_{X}<C_{S}^{-1}$. Here $C_{S}>0$ is an embedding constant which depends only on I determined by

$$
\|u\|_{C}+\left\|u_{x}\right\|_{C} \leq C_{S}\|u\|_{X} .
$$

Throughout this paper, the definition of the function spaces and their norms is presented in Section 2. In the second theorem, we derive the global existence of the sufficiently small solution in the norm of $X$. The conditions of $(\lambda, \delta)$ and $\left(u_{0}, u_{1}\right)$ are so complicated that we refer to the proof for more details.

Theorem 2. Let $\left(u_{0}, u_{1}\right) \in D$ with

$$
\left\|u_{0}\right\|_{X} \leq \frac{1-\theta}{C_{S}},
$$

for $0<\theta<1$. For sufficiently small $(\lambda, \delta)$ and small $\left(u_{0}, u_{1}\right)$, there exists a unique global solution of (1) with

$$
\|u(\cdot, t)\|_{X} \leq \frac{1-\theta}{C_{S}},
$$

and

$$
\phi=\left(\begin{array}{c}
u \\
u_{t}
\end{array}\right) \in C([0, \infty) ; D) \cap C^{1}([0, \infty) ; H) .
$$

The last theorem is on the quenching for sufficiently large $\lambda$.

Theorem 3. Let $\left(u_{0}, u_{1}\right) \in D$ with $u_{1}(x) \geq 0$ in I and

$$
\left\|u_{0}\right\|_{X} \leq \frac{1-\theta}{C_{S}}
$$

for $0<\theta<1$. For any $\lambda>\left(4 \pi^{2}\right) / 27$ and $\delta>0$, the solution of (1) quenches in finite time.

This paper is organized as follows: In Section 2, we transform (1) to the modified integral equation and apply the contraction mapping theorem to it so that we can obtain the local solution. In Section 3, we consider the global solution for the small parameter $(\lambda, \delta)$ and initial value $\left(u_{0}, u_{1}\right)$. In Section 4 , we prove that the solution quenches in finite time for the large 
parameter $\lambda$. We note that we do not have to impose any restriction on $\delta$. Hence this estimate is not optimal.

\section{Local solution}

We transform (1) to the modified Schrödinger equation as in [37, 38]. We apply the contraction mapping theorem owing to the facts for the Schrödinger equation in [1]. Then we establish the local solution. For $\delta=0$, the similar proof is done in [26, 31]. Different from Theorem 1 in [31], we truncate nonlinear term to deal with the derivative of $u(x)$. The proof is standard. However we prove it in order to see the effects of the fringing field $u_{x}^{2}$. In this paper, $C(\bar{I})$ denotes the space of all continuous functions in $\bar{I}$ with the norm

$$
\|u\|_{C}=\sup _{x \in \bar{I}}|u(x)|
$$

for $u \in C(\bar{I})$ and $W^{s, p}(I)$ denotes the usual Sobolev space in $I$ with the norm

$$
\|u\|_{W^{s, p}}=\left(\sum_{k=0}^{s}\left\|\frac{\partial^{k}}{\partial x^{k}} u\right\|_{p}^{p}\right)^{\frac{1}{p}}
$$

for $u \in W^{s, p}(I)$ with $s \in \mathbb{N}$ for $1 \leq p<\infty$ and

$$
\|u\|_{W^{s, \infty}}=\sum_{k=0}^{s}\left\|\frac{\partial^{k}}{\partial x^{k}} u\right\|_{\infty},
$$

for $u \in W^{s, \infty}(I)$ with $s \in \mathbb{N}$, respectively. Here, $\|\cdot\|_{p}$ denotes the standard $L^{p}$ norm in $I$ with $p \in[1, \infty]$. In particular, we denote $W^{s, 2}(I)=H^{s}(I) . H_{0}^{s}(I)$ is defined as the closure of the set $\mathscr{D}(I)$ in the space $H^{s}(I)$, where $\mathscr{D}(I)$ represents the space of all infinitely differentiable functions on $I$ with compact supports. Now the following Poincaré inequality holds:

$$
\|u\|_{2} \leq C_{P}\left\|u_{x}\right\|_{2}
$$

for $u \in H_{0}^{1}(I)$, where $C_{P}>0$ depends only on $I$. Hence we adopt the norm in $H_{0}^{1}(I)$ as

$$
\|u\|_{H_{0}^{1}} \equiv\left\|u_{x}\right\|_{2},
$$

for $u \in H_{0}^{1}(I)$ and in $X=H^{2}(I) \cap H_{0}^{1}(I)$ as

$$
\|u\|_{X} \equiv\left(\left\|u_{x x}\right\|_{2}^{2}+\left\|u_{x}\right\|_{2}^{2}\right)^{\frac{1}{2}}
$$

for $u \in X$, respectively. Since $I \subset \mathbb{R}$, we have the Sobolev embedding inequality

$$
\|u\|_{C}+\left\|u_{x}\right\|_{C} \leq C_{S}\|u\|_{X}
$$


for $u \in X$, where $C_{S}>0$ depends only on $I$. For the homogeneous damped wave equation

$$
\begin{cases}u_{t t}+u_{t}=u_{x x} & x \in I, t>0, \\ u(0, t)=u(1, t)=0 & t>0, \\ u(x, 0)=u_{0}(x) & x \in I, \\ u_{t}(x, 0)=u_{1}(x) & x \in I,\end{cases}
$$

we define

$$
\phi=\left(\begin{array}{l}
u \\
v
\end{array}\right)=\left(\begin{array}{c}
u \\
u_{t}
\end{array}\right) \text { and } A=i\left(\begin{array}{cc}
0 & i_{d} \\
-B^{2} & -i_{d}
\end{array}\right),
$$

where $i=\sqrt{-1}, B^{2}=-\partial^{2} /\left(\partial x^{2}\right)$ is a positive definite self-adjoint operator of $L^{2}(I)$ with domain $\mathscr{D}\left(B^{2}\right)=X=H^{2}(I) \cap H_{0}^{1}(I), \mathscr{D}(A)=D \subset H$ and $i_{d}$ denotes the identity operator on $H_{0}^{1}(I)$. Then, we can write (4) into the homogeneous Schrödinger equation

$$
\begin{cases}\phi_{t}=-i A \phi & x \in I, t>0, \\
\phi(0, t)=\phi(1, t)=0 & t>0, \\
\phi(x, 0)=\phi_{0}(x)=\left(\begin{array}{l}
u_{0}(x) \\
u_{1}(x)
\end{array}\right) & x \in I .\end{cases}
$$

Then we recall the following well-known results.

Lemma 1 (Cf. Théorèmes VII.4 et X.7 in [1]). For any $\phi_{0} \in D$, there exists a unique solution

$$
\phi \in C([0,+\infty) ; D) \cap C^{1}([0,+\infty) ; H),
$$

of (5). Moreover, we have

$$
\|\phi(\cdot, t)\|_{H} \leq\left\|\phi_{0}\right\|_{H}
$$

for $t>0$, where

$$
\|\phi\|_{H}=\left(\|u\|_{H_{0}^{1}}^{2}+\|v\|_{2}^{2}\right)^{\frac{1}{2}}
$$

Denoting the mapping $\exp (-i A t): D \rightarrow D$ defined by

$$
e^{-i A t} \phi_{0}(\cdot)=\phi(\cdot, t)
$$

then we have

$$
\left\|e^{-i A t}\right\| \leq M
$$


where $M>0$ depends only on $I$. For $0<\theta<1$, we define the modification $F_{\theta}(p)$ of $(1-p)^{-1}$ by

$$
F_{\theta}(p)= \begin{cases}\frac{1}{1-p} & \text { for } p \leq 1-\theta, \\ \frac{2}{\theta} & \text { for } p \geq 1-\theta / 2\end{cases}
$$

and extend $F_{\theta}$ suitably in the range $(1-\theta, 1-\theta / 2)$ so that $F_{\theta}$ and $F_{\theta}^{\prime}$ are uniformly Lipschitz continuous on $\mathbb{R}$ and $F_{\theta} \in W^{2, \infty}(\mathbb{R})$ with the Lipschitz constant $L_{1, \theta}$ and the following estimates

$$
\left\|F_{\theta}\right\|_{\infty} \leq L_{1, \theta}, \quad\left\|F_{\theta}^{\prime}\right\|_{\infty} \leq L_{1, \theta} \quad \text { and } \quad\left\|F_{\theta}^{\prime \prime}\right\|_{\infty} \leq L_{1, \theta},
$$

where $L_{1, \theta}>0$ depends only on $\theta$. Moreover we define the truncation $a_{\theta}(p)$ by

$$
a_{\theta}(p)= \begin{cases}1 & \text { for }|p| \leq 1-\theta \\ 0 & \text { for }|p| \geq 1-\theta / 2\end{cases}
$$

and extend $a_{\theta}$ suitably in the range $(\theta / 2-1, \theta-1)$ and $(1-\theta, 1-\theta / 2)$ so that $a_{\theta}$ and $a_{\theta}^{\prime}$ are uniformly Lipschitz continuous on $\mathbb{R}$ and $a_{\theta} \in W^{2, \infty}(\mathbb{R})$ with the Lipschitz constant $L_{2, \theta}$ and the following estimates

$$
\left\|a_{\theta}\right\|_{\infty} \leq L_{2, \theta}, \quad\left\|a_{\theta}^{\prime}\right\|_{\infty} \leq L_{2, \theta} \quad \text { and } \quad\left\|a_{\theta}^{\prime \prime}\right\|_{\infty} \leq L_{2, \theta},
$$

where $L_{2, \theta}>0$ depends only on $\theta$. Now let $L_{\theta}=\max \left(L_{1, \theta}, L_{2, \theta}\right)$. Denoting

$$
G_{\theta}\left(u, u_{x}\right)=a_{\theta}\left(u_{x}\right) F_{\theta}^{2}(u) u_{x}^{2},
$$

and

$$
J_{\theta}(u)=\left(\begin{array}{c}
0 \\
\lambda F_{\theta}^{2}(u)+\lambda \delta G_{\theta}\left(u, u_{x}\right)
\end{array}\right),
$$

we introduce the perturbed problem of (1) as

$$
\phi_{t}=-i A \phi+J_{\theta}(u)
$$

with the same initial and boundary conditions. Under these notations, we have the integral equation corresponding to perturbed problem

$$
\phi=e^{-i A t} \phi_{0}+\int_{0}^{t} e^{-i A(t-s)} J_{\theta}(u(s)) d s .
$$

Taking

$$
\eta \equiv\left\|\phi_{0}\right\|_{D}+1 \equiv\left(\left\|u_{0}\right\|_{X}^{2}+\left\|u_{1}\right\|_{H_{0}^{1}}^{2}\right)^{\frac{1}{2}}+1>0,
$$

we set

$$
X_{T} \equiv\left\{\phi \in C([0, T] ; D) \mid\|\phi\|_{X_{T}} \leq 2 \eta M\right\},
$$


where $T$ is a positive constant to be determined later. Here the space $X_{T}$ is equipped with the norm

$$
\|\phi\|_{X_{T}}=\sup _{t \in[0, T]}\|\phi(\cdot, t)\|_{D}=\sup _{t \in[0, T]}\left(\|u(\cdot, t)\|_{X}^{2}+\|v(\cdot, t)\|_{H_{0}^{1}}^{2}\right)^{\frac{1}{2}} .
$$

For $\phi \in X_{T}$, we define the mapping $S(t)$ by the right-hand side of (7), that is,

$$
S \phi=e^{-i A t} \phi_{0}+\int_{0}^{t} e^{-i A(t-s)} J_{\theta}(u(s)) d s .
$$

Then we show that $S$ is a contraction mapping from $X_{T}$ into $X_{T}$ for sufficiently small $T>0$.

Lemma 2. If

$$
T<T_{1} \equiv \frac{1}{2 \lambda L_{\theta}^{2} M}\left(2+5 \delta L_{\theta}\right)^{-1},
$$

then $S$ is a mapping from $X_{T}$ into $X_{T}$.

Proof. First of all, we have

$$
\left.\left|a_{\theta}(p)\right| p\right|^{k} \mid \leq\left\|a_{\theta}\right\|_{\infty} \leq L_{\theta} \quad \text { and }\left.\quad\left|a_{\theta}^{\prime}(p)\right| p\right|^{k} \mid \leq\left\|a_{\theta}^{\prime}\right\|_{\infty} \leq L_{\theta} \text {, }
$$

for $p \in \mathbb{R}$ and $k \in\{0\} \cup \mathbb{N}$. Let $\phi=\left(\begin{array}{l}u \\ v\end{array}\right) \in X_{T}$. Since we have

$$
\begin{aligned}
\left\|G_{\theta}\left(u, u_{x}\right)\right\|_{H_{0}^{1}} \leq & \left\|a_{\theta}^{\prime}\left(u_{x}\right) F_{\theta}^{2}(u) u_{x}^{2} u_{x x}\right\|_{2}+2\left\|a_{\theta}\left(u_{x}\right) F_{\theta}(u) F_{\theta}^{\prime}(u) u_{x}^{3}\right\|_{2} \\
& +2\left\|a_{\theta}\left(u_{x}\right) F_{\theta}^{2}(u) u_{x} u_{x x}\right\|_{2} \\
\leq & L_{\theta}^{3}\left\|u_{x x}\right\|_{2}+2 L_{\theta}^{3}\left\|u_{x}\right\|_{2}+2 L_{\theta}^{3}\left\|u_{x x}\right\|_{2} \\
\leq & 5 L_{\theta}^{3}\|u\|_{X},
\end{aligned}
$$

the following estimate holds:

$$
\begin{aligned}
\|S \phi\|_{D} & \leq M\left\|\phi_{0}\right\|_{D}+M \int_{0}^{t}\left\|J_{\theta}(u(s))\right\|_{D} d s \\
& \leq \eta M+\lambda M \int_{0}^{t}\left\|F_{\theta}^{2}(u)\right\|_{H_{0}^{1}} d s+\lambda \delta M \int_{0}^{t}\left\|G_{\theta}\left(u, u_{x}\right)\right\|_{H_{0}^{1}} d s \\
& \leq \eta M+2 \lambda L_{\theta}^{2} M \int_{0}^{t}\|u\|_{X} d s+5 \lambda \delta L_{\theta}^{3} M \int_{0}^{t}\|u\|_{X} d s \\
& \leq \eta M+2 \lambda \eta\left(2+5 \delta L_{\theta}\right) L_{\theta}^{2} M^{2} T,
\end{aligned}
$$

and

$$
\|S \phi\|_{X_{T}} \leq 2 \eta M
$$

Lemma 3. If $T<T_{2}$, then $S$ is a contraction mapping from $X_{T}$ into $X_{T}$, where $0<T_{2} \leq T_{1}$ and $T_{2}$ depends only on $\lambda, \delta, \theta, \eta$ and $I$. 
Proof. For any $\phi_{1}=\left(\begin{array}{c}u_{1} \\ v_{1}\end{array}\right) \in X_{T}$ and $\phi_{2}=\left(\begin{array}{c}u_{2} \\ v_{2}\end{array}\right) \in X_{T}$, we have

$$
\begin{aligned}
\left\|S \phi_{1}-S \phi_{2}\right\|_{D} \leq & \lambda M \int_{0}^{t}\left\|F_{\theta}^{2}\left(u_{1}\right)-F_{\theta}^{2}\left(u_{2}\right)\right\|_{H_{0}^{1}} d s \\
& +\lambda \delta M \int_{0}^{t}\left\|G_{\theta}\left(u_{1},\left(u_{1}\right)_{x}\right)-G_{\theta}\left(u_{2},\left(u_{2}\right)_{x}\right)\right\|_{H_{0}^{1}} d s .
\end{aligned}
$$

Through (3), (8) and uniformly Lipschitz continuity, the desired estimate is derived by the same computation as the proof of Lemma 2. For example, we have

$$
\begin{aligned}
& \left\|\left\{a_{\theta}^{\prime}\left(\left(u_{1}\right)_{x}\right)-a_{\theta}^{\prime}\left(\left(u_{2}\right)_{x}\right)\right\} F_{\theta}^{2}\left(u_{2}\right)\left(u_{2}\right)_{x}^{2}\left(u_{2}\right)_{x x}\right\|_{2} \\
& \leq L_{\theta}^{2}\left\|a_{\theta}^{\prime}\left(\left(u_{1}\right)_{x}\right)-a_{\theta}^{\prime}\left(\left(u_{2}\right)_{x}\right)\right\|_{\infty}\left\|\left(u_{2}\right)_{x}\right\|_{C}^{2}\left\|\left(u_{2}\right)_{x x}\right\|_{2} \\
& \leq C_{S}^{3} L_{\theta}^{3}\left\|u_{1}-u_{2}\right\|_{X}\left\|u_{2}\right\|_{X}^{3} \\
& \leq 8 \eta^{3} C_{S}^{3} L_{\theta}^{3} M^{3}\left\|\phi_{1}-\phi_{2}\right\|_{D},
\end{aligned}
$$

and deal with other terms similarly.

Proof of Theorem 1. By Lemmas 2 and 3, the mapping $S$ is a contraction mapping from $X_{T}$ to $X_{T}$ for sufficiently small $T \in\left(0, T_{2}\right)$. Hence (6) has a unique time local solution $\phi \in C([0, T) ; D)$. Since we get

$$
\begin{aligned}
\left\|v_{t}\right\|_{2} & \leq\|v\|_{2}+\left\|u_{x x}\right\|_{2}+\lambda\left\|F_{\theta}^{2}(u)\right\|_{2}+\lambda \delta\left\|G_{\theta}\left(u, u_{x}\right)\right\|_{2} \\
& \leq C_{P}\|v\|_{H_{0}^{1}}+\|u\|_{X}+\lambda L_{\theta}^{2}+\lambda \delta L_{\theta}^{3} \\
& \leq C_{P}\|v\|_{H_{0}^{1}}+\|u\|_{X}+\lambda L_{\theta}^{2}\left(1+\delta L_{\theta}\right)
\end{aligned}
$$

owing to (2) and (8), $\phi_{t} \in C([0, T) ; H)$ follows at once. If the solution of (6) begins with $\left\|u_{0}\right\|_{X} \leq$ $(1-\theta) / C_{S}$ and satisfies $\|u(\cdot, t)\|_{X} \leq(1-\theta) / C_{S}$ for all $t>0$, then we obtain

$$
\|u\|_{C}+\left\|u_{x}\right\|_{C} \leq C_{S}\|u\|_{X} \leq 1-\theta
$$

by (3), which implies that $u$ is a solution of (1). Otherwise there is a finite time $T_{0}>0$ at which $\left\|u\left(\cdot, T_{0}\right)\right\|_{X}=(1-\theta) / C_{S}$. We can choose $\theta_{1} \in(0, \theta)$ and apply the contraction mapping theorem to (6) with $\theta$ replaced by $\theta_{1}$. We may extend $u(x, t)$ uniquely to an interval $\left(0, T_{0}^{\prime}\right)$ with $T_{0}<T_{0}^{\prime}$ such that $\|u(\cdot, t)\|_{X} \leq\left(1-\theta_{1}\right) / C_{S}$ for $\left[0, T_{0}^{\prime}\right)$. Since we can take $\theta_{1} \in(0, \theta)$ arbitrarily small, $u(x, t)$ is a solution of (1) on $I \times\left[0, T_{0}^{\prime}\right)$ as long as $\|u(\cdot, t)\|_{X}<C_{S}^{-1}$.

Remark 1. Lemma 1 and (3) play an important role in the proof of Theorem 1. Hence under an appropriate setting, the method is applicable to a higher dimensional domain $\Omega$. For the sake of simplicity, we concentrate on the case $n=2,3$ and a bounded domain $\Omega \subset \mathbb{R}^{n}$ with 
smooth boundary $\partial \Omega$. In the proof, by modifying the construction of $F_{\theta}(p)$ and $a_{\theta}\left(q_{1}, q_{2}, \ldots, q_{n}\right)$ in order to satisfy

$$
F_{\theta} \in W^{3, \infty}(\mathbb{R}), \quad \text { and } \quad a_{\theta}\left(q_{1}, q_{2}, \ldots, q_{n}\right) \in W^{3, \infty}\left(\mathbb{R}^{n}\right),
$$

we have the following theorem.

Theorem 4. Assume that $\Omega$ is a bounded domain in $\mathbb{R}^{n}$ for $n=2,3$ with smooth boundary $\partial \Omega$. Let $X \equiv H^{3}(\Omega) \cap H_{0}^{1}(\Omega), Y \equiv H^{2}(\Omega) \cap H_{0}^{1}(\Omega), D \equiv X \times Y$ and $H \equiv Y \times H_{0}^{1}(\Omega)$. For any $\lambda>0, \delta>0$ and $\left(u_{0}, u_{1}\right) \in D$ with

$$
\left\|u_{0}\right\|_{X} \leq \frac{1-\theta}{C_{S}},
$$

for $0<\theta<1$, there exists a unique solution of (1) with

$$
\phi=\left(\begin{array}{c}
u \\
u_{t}
\end{array}\right) \in C([0, T) ; D) \cap C^{1}([0, T) ; H),
$$

for sufficiently small $T>0$. The solution $u$ can be continued as long as $\|u(\cdot, t)\|_{X}<C_{S}^{-1}$. Here $C_{S}>0$ is an embedding constant which depends only on $\Omega$ determined by

$$
\|u\|_{C}+\|\nabla u\|_{C} \leq C_{S}\|u\|_{X}
$$

\section{Global existence}

In Theorem 3.1 in [28], the authors establish necessary estimates for a nonlocal problem. We follow their method. As in Theorem 1 in this paper, we truncate the nonlinear term by $a_{\theta}\left(u_{x}\right)$ to deal with the estimate of $\|u\|_{X}$. On the other hand, we consider the case $\delta=0$ in Theorem 2 in [31] and utilize the energy function to derive the estimate of $\|u\|_{C}$. First of all, let begin with the fundamental lemma.

Lemma 4. Let $a, b \in \mathbb{R}$. The following inequalities hold:

$$
\begin{aligned}
& 0 \leq \frac{1}{2} a^{2}+a b+b^{2} . \\
& |a+2 b|^{2} \leq 2\left(a^{2}+4 b^{2}\right) \leq 24\left(\frac{1}{2} a^{2}+a b+b^{2}\right) . \\
& a^{2} \leq 4\left(\frac{1}{2} a^{2}+a b+b^{2}\right) . \\
& a^{2}+b^{2} \leq 6\left(\frac{1}{2} a^{2}+a b+b^{2}\right) .
\end{aligned}
$$


Proof of Theorem 2. We consider the modified problem

$$
u_{t t}+u_{t}=u_{x x}+\lambda F_{\theta}^{2}+\lambda \delta G_{\theta}
$$

where

$$
F_{\theta}=F_{\theta}(u) \text { and } G_{\theta}=G_{\theta}\left(u, u_{x}\right) .
$$

Multiplying this equation by $u$ and $u_{t}$, we have

$$
\frac{d}{d t} \int_{I}\left(u_{t} u+\frac{1}{2} u^{2}\right) d x+\int_{I}\left(-u_{t}^{2}+u_{x}^{2}\right) d x=\lambda \int_{I} u\left(F_{\theta}^{2}+\delta G_{\theta}\right) d x
$$

and

$$
\frac{d}{d t} \int_{I}\left(u_{t}^{2}+u_{x}^{2}\right) d x+2 \int_{I} u_{t}^{2} d x=2 \lambda \int_{I} u_{t}\left(F_{\theta}^{2}+\delta G_{\theta}\right) d x,
$$

respectively. Defining $\Phi(t)$ by

$$
\Phi(t)=\int_{I}\left(u_{t} u+\frac{1}{2} u^{2}+u_{t}^{2}+u_{x}^{2}\right) d x,
$$

we have from (2), (9) and Young's inequality

$$
0 \leq \Phi(t) \leq \frac{3}{2} \int_{I} u_{t}^{2} d x+\left(1+C_{P}^{2}\right) \int_{I} u_{x}^{2} d x \leq D \int_{I}\left(u_{t}^{2}+u_{x}^{2}\right) d x
$$

for $u \in X$ and $u_{t} \in H_{0}^{1}(I)$, where

$$
D=\max \left(\frac{3}{2}, 1+C_{P}^{2}\right)
$$

Hence adding these inequalities together, we obtain

$$
\begin{aligned}
\Phi^{\prime}+\frac{1}{D} \Phi & \leq \lambda \int_{I}\left|u+2 u_{t}\right| \cdot\left|F_{\theta}^{2}+\delta G_{\theta}\right| d x \\
& \leq \frac{1}{2}\left(\frac{1}{24 D} \int_{I}\left|u+2 u_{t}\right|^{2} d x+24 \lambda^{2} D \int_{I}\left|F_{\theta}^{2}+\delta G_{\theta}\right|^{2} d x\right) \\
& \leq \frac{1}{2 D} \Phi+24 \lambda^{2} D \int_{I} F_{\theta}^{4} d x+24 \lambda^{2} \delta^{2} D \int_{I} G_{\theta}^{2} d x \\
& \leq \frac{1}{2 D} \Phi+24 \lambda^{2} D L_{\theta}^{4}+24 \lambda^{2} \delta^{2} D L_{\theta}^{4} \int_{I} a_{\theta}^{2}\left(u_{x}\right) u_{x}^{4} d x \\
& \leq \frac{1}{2 D} \Phi+24 \lambda^{2} D L_{\theta}^{4}\left(1+\delta^{2} L_{\theta}^{2}\right),
\end{aligned}
$$

by (8), (10) and Young's inequality and finally

$$
\Phi^{\prime}+\frac{1}{2 D} \Phi \leq 24 \lambda^{2} D L_{\theta}^{4}\left(1+\delta^{2} L_{\theta}^{2}\right) .
$$

Multiplying this inequality by $\exp \left\{(2 D)^{-1} t\right\}$ and integrating it over $(0, t)$, we have

$$
\Phi(t) \leq e^{-\frac{1}{2 D} t} \Phi(0)+48 \lambda^{2} D^{2} L_{\theta}^{4}\left(1+\delta^{2} L_{\theta}^{2}\right) .
$$


Setting

$$
v=u_{t} \quad \text { and } \quad \Psi(t)=\int_{I}\left(v_{t} v+\frac{1}{2} v^{2}+v_{t}^{2}+v_{x}^{2}\right) d x
$$

we have

$$
\Psi^{\prime}+\frac{1}{D} \Psi \leq \lambda \int_{I}\left|v+2 v_{t}\right| \cdot\left|\left(F_{\theta}^{2}+\delta G_{\theta}\right)_{t}\right| d x,
$$

in the same way as $\Phi$ and $u$. Now the following estimate holds:

$$
\begin{aligned}
\left\|\left(F_{\theta}^{2}(u)+\delta G_{\theta}\left(u, u_{x}\right)\right)_{t}\right\|_{2} \leq & 2\left\|F_{\theta}(u) F_{\theta}^{\prime}(u) v\right\|_{2}+\delta\left\|a_{\theta}^{\prime}\left(u_{x}\right) F_{\theta}^{2}(u) u_{x}^{2} v_{x}\right\|_{2} \\
& +2 \delta\left\|a_{\theta}\left(u_{x}\right) F_{\theta}(u) F_{\theta}^{\prime}(u) u_{x}^{2} v\right\|_{2}+2 \delta\left\|a_{\theta}\left(u_{x}\right) F_{\theta}^{2}(u) u_{x} v_{x}\right\|_{2} \\
\leq & 2 L_{\theta}^{2}\|v\|_{2}+\delta L_{\theta}^{3}\left\|v_{x}\right\|_{2}+2 \delta L_{\theta}^{3}\|v\|_{2}+2 \delta L_{\theta}^{3}\left\|v_{x}\right\|_{2} \\
= & 2 L_{\theta}^{2}\left(1+\delta L_{\theta}\right)\|v\|_{2}+3 \delta L_{\theta}^{3}\left\|v_{x}\right\|_{2},
\end{aligned}
$$

and moreover

$$
\left\|\left(F_{\theta}^{2}(u)+\delta G_{\theta}\left(u, u_{x}\right)\right)_{t}\right\|_{2}^{2} \leq 32 L_{\theta}^{4}\left(1+\delta L_{\theta}\right)^{2} \Psi+18 \delta^{2} L_{\theta}^{6} \Psi \leq E \Psi,
$$

from (11), where $E=64 L_{\theta}^{4}+82 \delta^{2} L_{\theta}^{6}$. We multiply (13) by $\exp \left\{(2 D)^{-1} t\right\}$, utilize (10) and integrate it over $(0, t)$ to obtain

$$
\begin{aligned}
& \int_{0}^{t} e^{\frac{1}{2 D} s} \Psi^{\prime}(s) d s+\frac{1}{D} \int_{0}^{t} e^{\frac{1}{2 D} s} \Psi(s) d s \\
& \leq \frac{1}{2} \int_{0}^{t} e^{\frac{1}{2 D} s} d s\left(\frac{1}{48 D} \int_{I}\left|v+2 v_{t}\right|^{2} d x+48 \lambda^{2} D \int_{I}\left|\left(F_{\theta}^{2}+\delta G_{\theta}\right)_{t}\right|^{2} d x\right) \\
& \leq \frac{1}{4 D} \int_{0}^{t} e^{\frac{1}{2 D} s} \Psi(s) d s+24 \lambda^{2} D E \int_{0}^{t} e^{\frac{1}{2 D} s} \Psi d s \\
& =\left(\frac{1}{4 D}+1536 \lambda^{2} D L_{\theta}^{4}+1968 \lambda^{2} \delta^{2} D L_{\theta}^{6}\right) \int_{0}^{t} e^{\frac{1}{2 D} s} \Psi(s) d s \\
& <\frac{1}{2 D} \int_{0}^{t} e^{\frac{1}{2 D} s} \Psi(s) d s,
\end{aligned}
$$

as long as both

$$
\lambda^{2}<\frac{1}{12288 D^{2} L_{\theta}^{4}} \quad \text { and } \quad \lambda^{2} \delta^{2}<\frac{1}{15744 D^{2} L_{\theta}^{6}}
$$

Hence we have

$$
\Psi(t)<e^{-\frac{1}{2 D} t} \Psi(0)
$$

We conclude that

$$
\begin{aligned}
\|u\|_{X}^{2} & \leq 4\left\|v_{t}\right\|_{2}^{2}+4\|v\|_{2}^{2}+4 \lambda^{2}\left\|F_{\theta}^{2}(u)\right\|_{2}^{2}+4 \lambda^{2} \delta^{2}\left\|a_{\theta}\left(u_{x}\right) F_{\theta}^{2}(u) u_{x}^{2}\right\|_{2}^{2}+\left\|u_{x}\right\|_{2}^{2} \\
& \leq 24 \Psi(t)+4 \lambda^{2} L_{\theta}^{4}\left(1+\delta^{2} L_{\theta}^{2}\right)+\left\|u_{x}\right\|_{2}^{2} \\
& <24 e^{-\frac{1}{2 D} t} \Psi(0)+4 \lambda^{2} L_{\theta}^{4}\left(1+\delta^{2} L_{\theta}^{2}\right)+\Phi(t)
\end{aligned}
$$




$$
\begin{aligned}
& \leq 24 \Psi(0)+4 \lambda^{2} L_{\theta}^{4}\left(1+\delta^{2} L_{\theta}^{2}\right)+e^{-\frac{1}{2 D} t} \Phi(0)+48 \lambda^{2} D^{2} L_{\theta}^{4}\left(1+\delta^{2} L_{\theta}^{2}\right) \\
& \leq 24 \Psi(0)+\Phi(0)+4 \lambda^{2} L_{\theta}^{4}\left(1+12 D^{2}\right)\left(1+\delta^{2} L_{\theta}^{2}\right)
\end{aligned}
$$

thanks to (1), (8), (9) and (12) and that

$$
\|u\|_{X}^{2}<\frac{(1-\theta)^{2}}{C_{S}^{2}}
$$

if

$$
\begin{aligned}
\Psi(0) & <\frac{(1-\theta)^{2}}{96 C_{S}^{2}}, \quad \Phi(0)<\frac{(1-\theta)^{2}}{4 C_{S}^{2}}, \\
\lambda^{2} & <\min \left(\frac{1}{12288 D^{2} L_{\theta}^{4}}, \frac{(1-\theta)^{2}}{16 C_{S}^{2} L_{\theta}^{4}\left(1+12 D^{2}\right)}\right),
\end{aligned}
$$

and

$$
\lambda^{2} \delta^{2}<\min \left(\frac{1}{15744 D^{2} L_{\theta}^{6}}, \frac{(1-\theta)^{2}}{16 C_{S}^{2} L_{\theta}^{6}\left(1+12 D^{2}\right)}\right) .
$$

Remark 2. Since we have

$$
\Phi(0) \leq D\left(\left\|u_{0}\right\|_{H_{0}^{1}}^{2}+\left\|u_{1}\right\|_{2}^{2}\right)
$$

for instance, we choose

$$
\left\|u_{0}\right\|_{H_{0}^{1}} \leq \frac{1-\theta}{2 \sqrt{2} C_{S} \sqrt{D}} \quad \text { and } \quad\left\|u_{1}\right\|_{2} \leq \frac{1-\theta}{2 \sqrt{2} C_{S} \sqrt{D}},
$$

so that the condition

$$
\Phi(0)<\frac{(1-\theta)^{2}}{4 C_{S}^{2}}
$$

is achieved. In the same way, we obtain

$$
\begin{aligned}
\Psi(0) & \leq D\left(\left\|u_{1}\right\|_{H_{0}^{1}}^{2}+\left\|-u_{1}+\left(u_{0}\right)_{x x}+\lambda \frac{1+\delta\left(u_{0}\right)_{x}^{2}}{\left(1-u_{0}\right)^{2}}\right\|_{2}^{2}\right) \\
& \leq D\left(\left\|u_{1}\right\|_{H_{0}^{1}}^{2}+3\left\|u_{1}\right\|_{2}^{2}+3\left\|\left(u_{0}\right)_{x x}\right\|_{2}^{2}+3 \lambda^{2}\left\|\frac{1+\delta\left(u_{0}\right)_{x}^{2}}{\left(1-u_{0}\right)^{2}}\right\|_{2}^{2}\right) \\
& \leq\left(1+3 C_{P}^{2}\right) D\left\|u_{1}\right\|_{H_{0}^{1}}^{2}+3 D\left\|u_{0}\right\|_{X}^{2}+3 D \lambda^{2} \frac{\left(1+\delta C_{S}^{2}\left\|u_{0}\right\|_{X}^{2}\right)^{2}}{\left(1-C_{S}\left\|u_{0}\right\|_{X}\right)^{4}} \\
& \leq\left(1+3 C_{P}^{2}\right) D\left\|u_{1}\right\|_{H_{0}^{1}}^{2}+3 D\left\|u_{0}\right\|_{X}^{2}+3 D \lambda^{2} \frac{\left\{1+\delta(1-\theta)^{2}\right\}^{2}}{\theta^{4}} .
\end{aligned}
$$

Hence we can take $\left(u_{0}, u_{1}\right)$ and $(\lambda, \delta)$ so as to satisfy

$$
\Psi(0)<\frac{(1-\theta)^{2}}{96 C_{S}^{2}} .
$$


Remark 3. To extend the result in Theorem 2 to a higher dimensional domain $\Omega \subset \mathbb{R}^{n}$ for $n=2,3$, we estimate $\|u\|_{H^{3}}$ owing to the inclusion

$$
H^{3}(\Omega) \subset C^{1}(\bar{\Omega})
$$

To achieve this aim, we have to consider, for instance,

$$
X(t)=\int_{\Omega}\left(\nabla v_{t} \cdot \nabla v+\frac{1}{2}|\nabla v|^{2}+\left|\nabla v_{t}\right|^{2}+(\Delta v)^{2}\right) d x,
$$

in addition to $\Phi(t)$ and $\Psi(t)$ in the proof of Theorem 2. However, it is difficult to control the term $\||\nabla \nu| \Delta u\|_{2}$ which appears from $X^{\prime}(t)$. Hence, the extension to $n>1$ seems to be open.

\section{Quenching}

We apply Kaplan's method in [21]. The estimates in our proof are quite similar to those in $[24,32]$.

Proof of Theorem 3. For the first positive eigenvalue $\mu=\pi^{2}$ of $-d^{2} /\left(d x^{2}\right)$, let $\psi=(\pi / 2) \sin \pi x$ be the corresponding eigenfunction i.e.,

$$
\begin{cases}-\psi_{x x}=\mu \psi & x \in I, \\ \psi(0)=\psi(1)=0, & \\ \psi>0 & x \in I .\end{cases}
$$

Then we have $\int_{I} \psi d x=1$. We set

$$
z(t)=\int_{I} u(x, t) \psi(x) d x .
$$

Differentiating $z(t)$ twice, we obtain

$$
z_{t t}+z_{t}=-\mu z+\lambda \int_{I} \frac{1+\delta u_{x}^{2}}{(1-u)^{2}} \psi d x \geq-\mu z+\lambda \int_{I} \frac{\psi}{(1-u)^{2}} d x
$$

Since the Jensen inequality [41] implies that

$$
\int_{I} \frac{\psi}{(1-u)^{2}} d x \geq \frac{1}{(1-z)^{2}},
$$

we have

$$
z_{t t}+z_{t} \geq\left\{\lambda-\mu z(1-z)^{2}\right\} \frac{1}{(1-z)^{2}}
$$

Now we assume that

$$
u(x, t)<1
$$


for $x \in I$ and $t \geq 0$. Then we have

$$
z(t) \leq \int_{I} \psi(x) d x=1
$$

A simple calculation yields

$$
z(1-z)^{2} \leq \frac{4}{27}
$$

for $z \leq 1$. Hence we get

$$
z_{t t}+z_{t} \geq\left(\lambda-\frac{4 \mu}{27}\right) \frac{1}{(1-z)^{2}} .
$$

Multiplying this inequality by exp $t$ and integrating it over $(0, t)$, we have

$$
z_{t} \geq e^{-t} z_{t}(0)+\left(\lambda-\frac{4 \mu}{27}\right) e^{-t} \int_{0}^{t} \frac{e^{s}}{(1-z(s))^{2}} d s .
$$

By the assumption, we have

$$
z_{t}(0)=\int_{I} u_{1}(x) \psi(x) d x \geq 0,
$$

and hence $z_{t}(t)>0$ for $t>0$ with $\lambda>(4 \mu) / 27$. Thus by $0 \leq z(0)<z(t) \leq 1$, we have

$$
z_{t} \geq e^{-t} z_{t}(0)+\left(\lambda-\frac{4 \mu}{27}\right) \frac{1}{(1-z(0))^{2}}\left(1-e^{-t}\right),
$$

and

$$
z(t) \geq z(0)+\left(1-e^{-t}\right) z_{t}(0)+\left(\lambda-\frac{4 \mu}{27}\right) \frac{1}{(1-z(0))^{2}}\left(t+e^{-t}-1\right),
$$

by integrating it over $(0, t)$. Since we have

$$
\lim _{t \rightarrow+\infty}\left(t+e^{-t}-1\right)=+\infty \text { and } 1-e^{-t} \in[0,1),
$$

for $t \geq 0$, we conclude that

$$
\max _{x \in \bar{I}} u(x, t) \geq \int_{I} u(x, t) \psi(x) d x=z(t) \geq 1,
$$

for sufficiently large $t>0$, which leads us to a contradiction.

Remark 4. In [39, 40], the authors consider the stationary problem of (1) given by

$$
\begin{cases}\Delta u+\lambda \frac{1+\delta|\nabla u|^{2}}{(1-u)^{2}}= & x \in \Omega \\ u(x)=0 & x \in \partial \Omega\end{cases}
$$

Assume that $\Omega$ is a bounded domain in $\mathbb{R}^{n}$ for $n \in \mathbb{N}$ with smooth boundary $\partial \Omega$. For fixed $\delta>0$, they find a pull-in voltage $\lambda_{\delta}^{*}$ such that there exists a minimal solution $v$ for $\lambda<\lambda_{\delta}^{*}$ and there is no solution for $\lambda>\lambda_{\delta}^{*}$. Moreover in [30, 39], the authors consider the parabolic problem

$$
u_{t}=\Delta u+\lambda \frac{1+\delta|\nabla u|^{2}}{(1-u)^{2}}
$$


instead of (1). Thanks to the maximal principle, they show that the solution exists globally in time for $\lambda \leq \lambda_{\delta}^{*}$ and that quenches in finite time for $\lambda>\lambda_{\delta}^{*}$. In our case, $\lambda_{\delta}^{*} \leq\left(4 \pi^{2}\right) / 27$ holds. However, it is not clear that $\lambda_{\delta}^{*}=\left(4 \pi^{2}\right) / 27$.

The proof is applicable to a higher dimensional domain $\Omega$. We have the following:

Theorem 5. Under the same assumptions in Theorem 4. Let $\left(u_{0}, u_{1}\right) \in D$ with $u_{1}(x) \geq 0$ in $\Omega$ and

$$
\left\|u_{0}\right\|_{X} \leq \frac{1-\theta}{C_{S}}
$$

for $0<\theta<1$. If $\lambda>(4 \mu) / 27$, then the solution of (1) quenches in finite time, where $\mu$ is the first positive eigenvalue of $-\Delta$.

\section{Acknowledgement}

The author would like to express his deepest gratitude to the referee for useful suggestions on the publication.

\section{References}

[1] H. Brezis, Analyse fonctionnelle, Masson, Paris, 1983.

[2] C. Y. Chan and K. K. Nip, On the blow-up of $\left|u_{t t}\right|$ at quenching for semilinear Euler-Poisson-Darboux equations, Mat. Apl. Comput., 14 (1995), 185-190.

[3] P. H. Chang and H. A. Levine, The quenching of solutions of semilinear hyperbolic equations, SIAM J. Math. Anal., 12 (1981), 893-903.

[4] J. Dávila and J. Wei, Point ruptures for a MEMS equation with fringing field, Comm. Partial Differential Equations, 37 (2012), 1462-1493.

[5] P. Esposito, Compactness of a nonlinear eigenvalue problem with a singular nonlinearity, Commun. Contemp. Math., 10 (2008), 17-45.

[6] P. Esposito and N. Ghoussoub, Uniqueness of solutions for an elliptic equation modeling MEMS, Methods Appl. Anal., 15 (2008), 341-353.

[7] P. Esposito, N. Ghoussoub and Y. Guo, Compactness along the branch of semistable and unstable solutions for an elliptic problem with a singular nonlinearity, Comm. Pure Appl. Math., 60 (2007), 1731-1768.

[8] P. Esposito, N. Ghoussoub and Y. Guo, Mathematical analysis of partial differential equations modeling electrostatic MEMS, Courant Lecture Notes in Mathematics, 20, Courant Institute of Mathematical Sciences, New York; American Mathematical Society, Providence, RI, 2010.

[9] G. Flores, G. Mercado, J. A. Pelesko and N. Smyth, Analysis of the dynamics and touchdown in a model of electrostatic MEMS, SIAM J. Appl. Math., 67 (2006/07), 434-446.

[10] M. Fukunishi and T. Watanabe, Variational approach to MEMS model with fringing field, J. Math. Anal. Appl., 423 (2015), 1262-1283.

[11] N. Ghoussoub and Y. Guo, On the partial differential equations of electrostatic MEMS devices: stationary case, SIAM J. Math. Anal., 38 (2006/07), 1423-1449.

[12] N. Ghoussoub and Y. Guo, On the partial differential equations of electrostatic MEMS devices. II. Dynamic case, NoDEA Nonlinear Differential Equations Appl., 15 (2008), 115-145. 
[13] J.-S. Guo, Quenching problem in nonhomogeneous media, Differential Integral Equations, 10 (1997), 10651074 .

[14] J.-S. Guo, B. Hu and C.-J. Wang, A nonlocal quenching problem arising in a micro-electro mechanical system, Quart. Appl. Math., 67 (2009), 725-734.

[15] J.-S. Guo and B.-C. Huang, Hyperbolic quenching problem with damping in the micro-electro mechanical system device, Discrete Contin. Dyn. Syst. Ser. B, 19 (2014), 419-434.

[16] J.-S. Guo and N. I. Kavallaris, On a nonlocal parabolic problem arising in electrostatic MEMS control, Discrete Contin. Dyn. Syst., 32 (2012), 1723-1746.

[17] J.-S. Guo and P. Souplet, No touchdown at zero points of the permittivity profile for the MEMS problem, SIAM J. Math. Anal., 47 (2015), 614-625.

[18] Y. Guo, On the partial differential equations of electrostatic MEMS devices. III. Refined touchdown behavior, J. Differential Equations, 244 (2008), 2277-2309.

[19] Y. Guo, Global solutions of singular parabolic equations arising from electrostatic MEMS, J. Differential Equations, 245 (2008), 809-844.

[20] K. M. Hui, The existence and dynamic properties of a parabolic nonlocal MEMS equation, Nonlinear Anal., 74 (2011), 298-316.

[21] S. Kaplan, On the growth of solutions of quasi-linear parabolic equations, Comm. Pure Appl. Math., 16 (1963), 305-330.

[22] N. I. Kavallaris, A. A. Lacey, C. V. Nikolopoulos and D. E. Tzanetis, A hyperbolic non-local problem modelling MEMS technology, Rocky Mountain J. Math., 41 (2011), 505-534.

[23] N. I. Kavallaris, T. Miyasita and T. Suzuki, Touchdown and related problems in electrostatic MEMS device equation, NoDEA Nonlinear Differential Equations Appl., 15 (2008), 363-385.

[24] P. Laurençot and C. Walker, A fourth-order model for MEMS with clamped boundary conditions, Proc. Lond. Math. Soc. (3), 109 (2014), 1435-1464.

[25] H. A. Levine, Quenching, nonquenching, and beyond quenching for solution of some parabolic equations, Ann. Mat. Pura Appl., 155 (1989), 243-260.

[26] H. A. Levine and M. W. Smiley, Abstract wave equations with a singular nonlinear forcing term, J. Math. Anal. Appl., 103 (1984), 409-427.

[27] C. Liang, J. Li and K. Zhang, On a hyperbolic equation arising in electrostatic MEMS, J. Differential Equations, 256 (2014), 503-530.

[28] C. Liang and K. Zhang, Global solution of the initial boundary value problem to a hyperbolic nonlocal MEMS equation, Comput. Math. Appl., 67 (2014), 549-554.

[29] A. E. Lindsay and M. J. Ward, Asymptotics of some nonlinear eigenvalue problems for a MEMS capacitor. I. Fold point asymptotics, Methods Appl. Anal., 15 (2008), 297-325.

[30] Z. Liu and X. Wang, On a parabolic equation in MEMS with fringing field, Arch. Math. (Basel), 98 (2012), 373-381.

[31] T. Miyasita, Global existence of radial solutions of a hyperbolic MEMS equation with nonlocal term, Differ. Equ. Appl., 7 (2015), 169-186.

[32] F. K. N'gohisse and T. K. Boni, Quenching time of some nonlinear wave equations, Arch. Math. (Brno), 45 (2009), 115-124.

[33] J. A. Pelesko, Mathematical modeling of electrostatic MEMS with tailored dielectric properties, SIAM J. Appl. Math., 62 (2001/02), 888-908.

[34] J. A. Pelesko and D. H. Bernstein, Modeling MEMS and NEMS, Chapman \& Hall/CRC, Boca Raton, FL, 2003.

[35] J. A. Pelesko and T. A. Driscoll, The effect of the small-aspect-ratio approximation on canonical electrostatic MEMS models, J. Engrg. Math., 53 (2005), 239-252.

[36] J. A. Pelesko and A. A. Triolo, Nonlocal problems in MEMS device control, J. Engrg. Math., 41 (2001), 345-366.

[37] M. Reed, Abstract non-linear wave equations, Lecture Notes in Mathematics, 507, Springer-Verlag, BerlinNew York, 1976.

[38] R. A. Smith, On a hyperbolic quenching problem in several dimensions, SIAM J. Math. Anal., 20 (1989), 10811094. 
[39] Q. Wang, Estimates for the quenching time of a MEMS equation with fringing field, J. Math. Anal. Appl., 405 (2013), 135-147.

[40] J. Wei and D. Ye, On MEMS equation with fringing field, Proc. Amer. Math. Soc., 138 (2010), $1693-1699$.

[41] S. Zheng, Nonlinear parabolic equations and hyperbolic-parabolic coupled systems, Pitman Monographs and Surveys in Pure and Applied Mathematics, 76, Longman Scientific \& Technical, Harlow; copublished in the United States with John Wiley \& Sons, Inc., New York, 1995.

250-201 Imamichi-cho, Kyoto 605-0042, Japan.

E-mail: sk109685@mail.doshisha.ac.jp 